\title{
Elastic scattering studies of aligned DMPC multilayers on different hydrations ${ }^{1}$
}

\author{
Marcus Trapp a , Fanni Juranyi ${ }^{\text {b }}$, Moeava Tehei ${ }^{\text {c,d }}$, Lambert van Eijck ${ }^{\mathrm{e}}$, Bruno Demé ${ }^{\mathrm{e}}$, \\ Thomas Gutberlet ${ }^{\mathrm{f}}$ and Judith Peters ${ }^{\mathrm{a}, \mathrm{e}, \mathrm{g}, *}$ \\ ${ }^{a}$ Institut de Biologie Structurale J.-P. Ebel, UMR 5075, CNRS-CEA-UJF, Grenoble, France \\ ${ }^{\mathrm{b}}$ LNS, ETH Zürich and Paul Scherrer Institut, Villigen, Switzerland \\ ${ }^{\mathrm{c}}$ Australian Institute of Nuclear Science and Engineering, Menai, Australia \\ ${ }^{\mathrm{d}}$ School of Chemistry and Centre for Medical Bioscience, University of Wollongong, Wollongong, \\ Australia \\ ${ }^{\mathrm{e}}$ Institut Laue-Langevin, Grenoble, France \\ ${ }^{\mathrm{f}}$ JCNS at FRMII, Garching, Germany \\ ${ }^{\mathrm{g}}$ Université Joseph Fourier, Grenoble, France
}

\begin{abstract}
Biological membranes, consisting mainly of phospholipids and proteins, are organized in a bilayered structure which exhibits dynamical behaviour within time regimes ranging from $10^{-12} \mathrm{~s}$ with the motion of alkyl chain defects and $1 \mathrm{~s}$ corresponding to collective excitations of the bilayer [Europhysics Letters 8 (1989), 201-206]. With the prominent role hydration plays on the structural phase behaviour of phospholipids membranes, it is essential for a better description of membranes to understand also the influence of hydration on the dynamics of membrane systems. In the present study we have performed neutron scattering investigations on highly oriented DMPC-d54 multilayers at two different relative humidity (rh) levels. Our results reveal the strong influence of hydration on the local membrane dynamics, i.e., head group dynamics.
\end{abstract}

Keywords: Oriented model membranes, elastic neutron scattering, dynamics, hydration effects

\section{Introduction}

Biological membranes consist not only of different kinds of lipids, but also of membrane proteins and molecules like, e.g., cholesterol and ethanol [18]. To investigate the physical and structural behaviour of such complex systems, lipid membranes consisting of only one type of lipid such as 1,2-Dimyristoyl$s n$-Glycero-3-Phosphocholine (DMPC) serve as suitable model systems.

Saturated phospholipids like DMPC show two phase transitions: (1) a pre-transition from the $\mathrm{L}_{\beta}$ gel phase to the $\mathrm{P}_{\beta}$ ripple phase (DMPC at $286 \mathrm{~K}$ ) and (2) the main phase transition from the $\mathrm{P}_{\beta}$ ripple phase to the liquid-crystalline $\mathrm{L}_{\alpha}$ phase (DMPC at $296 \mathrm{~K}$ ) where the mobility of the alkyl chains is enhanced with respect to the gel phase [10]. For chain deuterated lipids which were used in this study the temperature of the main phase transition is shifted by about $3 \mathrm{~K}$ to lower temperatures [6].

Lowering the water content reduces the repeating distance of the bilayers. In addition, structural investigations [20] show that a lower degree of hydration causes a shift of the main phase transition to

\footnotetext{
${ }^{1}$ This paper was presented at ECSBM, Palermo, Italy, August 28-September 2, 2009. Guest Editor: A. Cupane.

*Corresponding author: Judith Peters, Université Joseph Fourier, F-38042 Grenoble Cédex 9, France. Tel.: +33 4762075 60; Fax: +33 4762076 88; E-mail: peters@ill.fr.
} 
higher temperatures. The effect can be observed by X-ray or neutron diffraction following the shift in the distance of the Bragg peaks of the lipid bilayers. The relation $d=\lambda / 2 \times \sin (\theta)(\lambda-\mathrm{X}$-ray or neutron wavelength, $\theta$ - scattering angle) allows to calculate the repeating distance $d$. For a fully hydrated DMPC bilayer, the repeating distance $d$ is around $63 \AA$ [8]. While the influence of hydration on the structural properties of membranes is well investigated [20], its influence on dynamic behaviour is rather poor.

The diffusive motions of lipids within the bilayer have been explored by quasi-elastic neutron scattering [7,14,22]. More recently collective motions of the lipid bilayer were also studied with inelastic neutron scattering [15]. However, the hydration effects on the lipid bilayer properties were not enclosed in these investigations. In this study, we thus performed neutron scattering investigations on highly oriented DMPC multilayers at two different relative humidity (rh) levels with the aim to fill this gap. Our results reveal the strong influence of hydration on the local membrane dynamics.

\section{Materials and methods}

\subsection{Sample preparation}

To probe dynamics of lipid membranes in and out of plane, oriented samples can be prepared on very smooth surfaces such as silicon wafers or mica sheets. Solid supported bilayer systems also allow the preparation of large amount of sample $(\approx 150-200 \mathrm{mg})$ required in inelastic and quasi-elastic neutron scattering experiments [19]. Another advantage of this preparation method is the very low mosaic spread (below $0.5^{\circ}$ ). Each $\mathrm{Si}$-wafer was of size of about $30 \times 40 \mathrm{~mm}$ which fitted perfectly the dimensions of the flat gold coated aluminium sample cells used for the experiments.

Alkyl chain deuterated 1,2-dimyristoylphosphatidylcholine-d54 (DMPC-d54) was purchased from Avanti Polar Lipids (Alabaster, AL, USA) and used without further purification. The DMPC powder was dissolved in a 3:1 chloroform-trifluoroethanol (TFE) mixture following a protocol described in [2]. This solution was then kept at $-20^{\circ} \mathrm{C}$ overnight.

About $30 \mathrm{mg}$ of lipid was deposited on a single wafer. After the deposition the wafers were dried for two days in a desiccator. One sample was rehydrated from pure $\mathrm{D}_{2} \mathrm{O}$ with relative humidity of $100 \%$, another one was rehydrated from a saturated salt solution $\left(\mathrm{D}_{2} \mathrm{O}+\mathrm{NaCl}\right)$ with reduced relative humidity of $75 \%$. Six such wafers were placed in the sample cell and sealed to achieve an amount of about $200 \mathrm{mg}$ hydrated lipid bilayers per sample. The weight of both samples was monitored and no change in weight before and after the neutron experiments was observed.

\subsection{Elastic neutron experiments}

The level of hydration, the mosaicity of the 1D lamellar order and the evolution of the repeat distance (d-spacing) of the bilayers with temperature were checked by neutron diffraction measurements on the small momentum transfer diffractometer D16 at the Institut Laue-Langevin (ILL), Grenoble, France [1]. After heating to $330 \mathrm{~K}$ to allow annealing of defects, scans were performed to obtain the repeat distance of the membranes at three different temperatures, namely, 330, 310 and $280 \mathrm{~K}$. During the temperature changes data were taken to identify the temperature of the main phase transition. An example of the evolution of the d-spacing with temperature is shown in Fig. 1.

Elastic temperature scans in the range of $280-330 \mathrm{~K}$ were performed on the cold neutron backscattering spectrometer IN16 at ILL at an energy resolution of $\Delta E=0.9 \mu \mathrm{eV}$ (full width half maximum) and an accessible $Q$-range of $0.19-1.93 \AA^{-1}$ [5]. The investigated temperature range covers both the main 


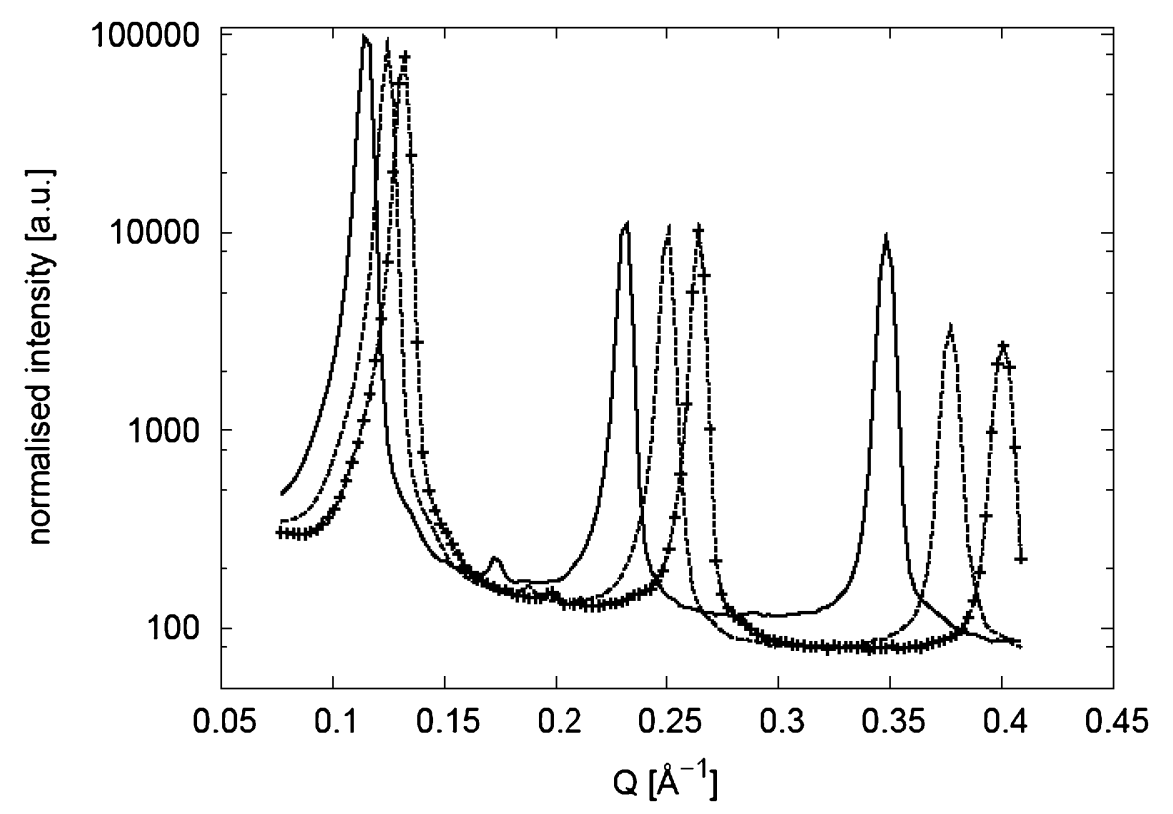

Fig. 1. Diffraction data taken on D16 at ILL. Data are shown for three different temperatures: $280 \mathrm{~K}$ (straight line), $310 \mathrm{~K}$ (broken line) and $330 \mathrm{~K}$ (broken line with crosses). The sample was hydrated from a saturated salt solution $\left(\mathrm{D}_{2} \mathrm{O}+\mathrm{NaCl}\right)$ resulting in a relative humidity of $75 \%$.

phase transition from the $\mathrm{P}_{\beta}$ ripple to the liquid-crystalline $\mathrm{L}_{\alpha}$ phase which occurs around $296 \mathrm{~K}$ for DMPC at full hydration and also the pre-transition from the $\mathrm{L}_{\beta}$ gel phase to the $\mathrm{P}_{\beta}$ ripple phase about 10 degrees below the main phase transition.

Special care was taken on the orientation of the sample to probe motions around the chain correlation peak at $Q=1.48 \AA^{-1}$ parallel and perpendicular to the membrane surface. Via the relation $Q=$ $4 \pi \sin (2 \theta) / \lambda$ the angle of the sample with respect to the incoming beam was calculated to be $135^{\circ}$ for the orientation parallel to the membrane surface and $45^{\circ}$ for the perpendicular orientation, respectively. From the obtained data an empty cell was subtracted and the data were normalised by the scattering of a $1 \mathrm{~mm}$ vanadium sample.

\section{Results and discussion}

From the shift of the main phase transition temperature to higher temperatures with decreasing water content (see Section 1 and Fig. 1 in [20]), it is possible to evaluate the water content between the phospholipid surfaces. We can extract the parameter $R_{\mathrm{w}}=n_{\mathrm{W}} / n_{\mathrm{A}}$ where $R_{\mathrm{w}}$ expresses the molar ratio of water $\left(n_{\mathrm{W}}\right)$ and amphiphile $\left(n_{\mathrm{A}}\right)$ [12,13]. Figure 2 (using the left $y$-axis) shows the summed elastic intensities measured for the parallel orientation at $135^{\circ}$ with respect to the incoming beam for the two samples on IN16. At the temperature of the main phase transition a drastic decrease in the elastic intensity occurs. The phase transition temperature moves to higher temperatures for the sample with lower water content. The calculated $R_{\mathrm{w}}$ for the higher hydrated sample is $R_{\mathrm{w}} \approx 6$ (transition temperature: $305 \mathrm{~K}$ ) and $R_{\mathrm{w}} \approx 4$ for the less hydrated sample (transition temperature: $310 \mathrm{~K}$ ). Pfeiffer et al. find for DMPC multilayers a value of $R_{\mathrm{w}} \geqslant 12$ for fully hydrated membranes [13]. 


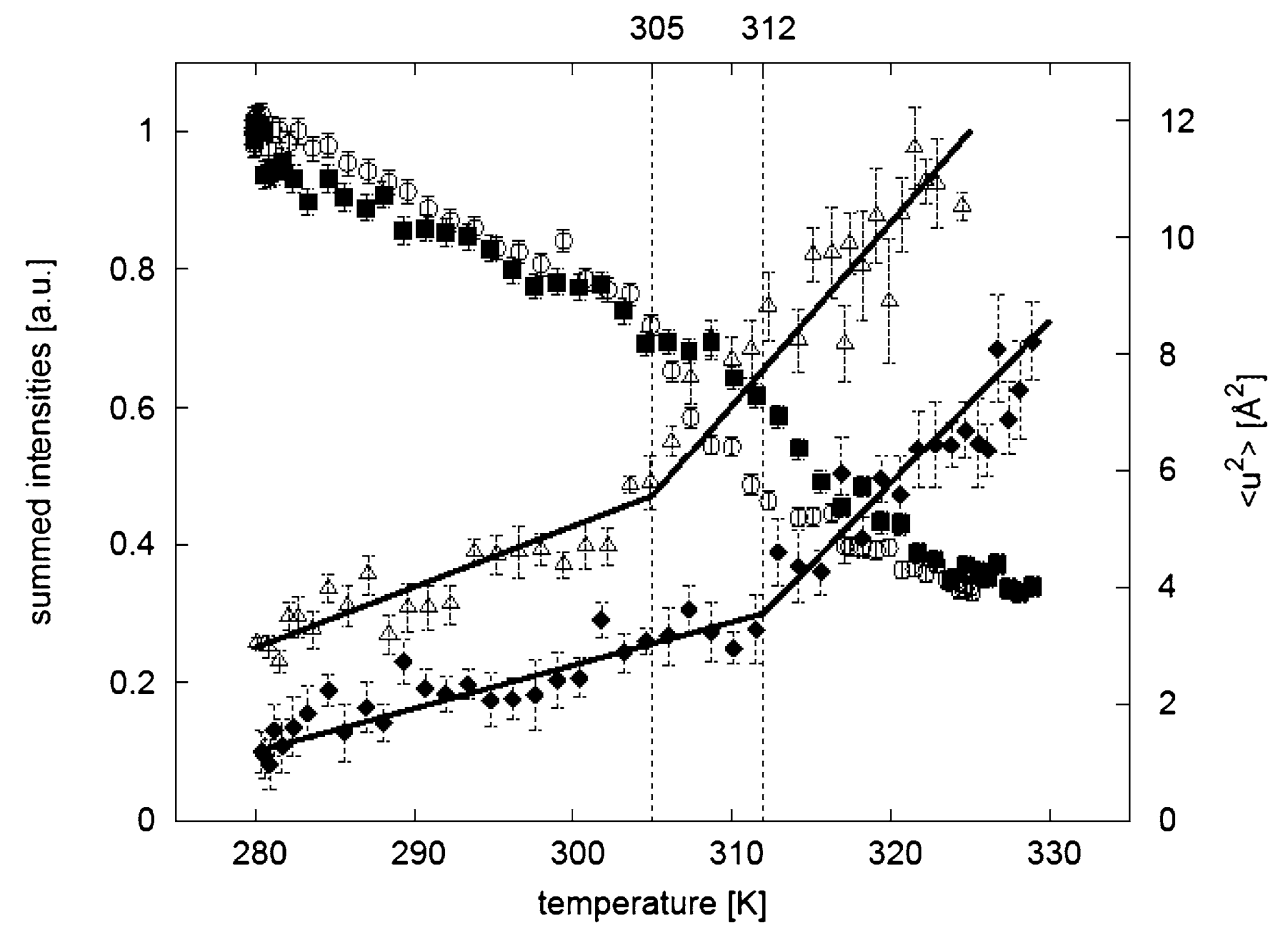

Fig. 2. Summed elastic intensity ( $Q$-range: $0.43-1.16 \AA^{-1}$ ) measured at $135^{\circ}$ (left $y$-scale, filled squares: $R_{\mathrm{W}} \approx 4$, empty circles: $R_{\mathrm{W}} \approx 6$ ) and mean square displacements $\left\langle u^{2}\right\rangle$ (right $y$-scale, filled diamonds: $R_{\mathrm{W}} \approx 4$, empty triangles: $R_{\mathrm{W}} \approx 6$ ). Drawn lines are guides to the eyes to follow the evolution of the mean square displacements. Data taken on the cold neutron spectrometer IN16 at ILL (energy resolution: $0.9 \mu \mathrm{eV}$ ). Intensities are normalised to the lowest temperature.

To characterise the local dynamics from the elastic neutron scattering intensity, the so-called mean square displacements (msd) $\left\langle u^{2}\right\rangle$ were calculated. The elastic scattering function $S(Q, \omega=0)$ can be approximated by $S(Q, \omega=0) \approx \exp \left(1 / 6 \times\left\langle u^{2}\right\rangle Q^{2}\right)$ [21]. From the slope of a linear fit to a semilogarithmic plot of the data one can extract the $\left\langle u^{2}\right\rangle$. Below the temperature of the main phase transition, coherent scattering arising from the ordering of the alkyl chain, gives rise to the so-called "chain correlation peak" around a characteristic $Q$-value of $1.48 \AA^{-1}$. Therefore the fit range was limited to $0.18 \AA^{-2} \leqslant Q^{2} \leqslant 1.33 \AA^{-2}$ in our data analysis. As shown in Fig. 2 (using the right $y$-axis) the $\left\langle u^{2}\right\rangle$ show transitions at 305 and $310 \mathrm{~K}$ for the higher and lower hydrated sample, respectively. At first sight the evaluated mean square displacements are very large above temperature of the main phase transition, but $\left\langle u^{2}\right\rangle$ in the same order of magnitude have been observed in a previous study which aimed to investigate the influence of the myelin basic protein (MBP) on the dynamics of model membranes (DMPA in this study) [11]. A recently published molecular-dynamics simulation performed on fully hydrated DMPC bilayers in the $\mathrm{L}_{\alpha}$ phase (at $303 \mathrm{~K}$ ) also shows very large $\left\langle u^{2}\right\rangle$ at this temperature, even larger then the two partially hydrated samples used in our study [4]. Hence we attribute the difference in the values of the mean square displacements to the different hydrations of the samples, ranging from the highest $\left\langle u^{2}\right\rangle$-value for the simulation $\left(\approx 8 \AA^{2}\right.$ for the centre of mass) to the sample with the lowest hydration of $R_{\mathrm{w}} \approx 4\left(\approx 2.9 \AA^{2}\right)$. The authors differentiate three different diffusion regimes in time: (1) a ballistic region where $\left\langle u^{2}\right\rangle \sim t^{2}$; (2) a subdiffusive domain where $\left\langle u^{2}\right\rangle \sim t^{\beta}$ with $\beta<1$ and (3) a domain of Fickian diffusion with $\left\langle u^{2}\right\rangle \sim t$. According to this simulation, the time window of IN16 of about 700 picoseconds probes length scales in the subdiffusive regime. A detailed analysis of quasi- 
elastic data taken in both the $\mathrm{L}_{\beta}$ gel and liquid-crystalline $\mathrm{L}_{\alpha}$ phase is still in progress [23]. In order to compare the results obtained from the mean square displacement (using the right $y$-axis in Fig. 2) with the summed elastic intensities, the intensities shown in Fig. 2 (using the left $y$-axis) were summed over the same $Q$-range $\left(0.43 \AA^{-1} \leqslant Q \leqslant 1.16 \AA^{-1}\right)$ which was used in the evaluation of the msd's. The effect is stronger for the summed intensities due to adding up the signals from all detectors in the used $Q$-range. From hydration dependent studies of, e.g., bacteriorhodopsin [9] it is known that with increasing hydration the msd's show a steeper slope only at high relative humilities in contrast to protein where a steady rice of the msd is observed [17]. Only small difference of the calculated slopes in the $\mathrm{L}_{\alpha}$ phase (higher hydrated sample: $0.258 \pm 0.0157$, less hydrated sample: $0.224 \pm 0.022$ ) of our results confirm these finding.

Rheinstaedter et al. [16] analyzed the elastic intensity at various $Q$-values of DMPC to map out the transition of the different molecular components from immobile to mobile as a function of temperature. The effect of hydration on the membrane dynamics was neglected in their analysis. Our study reveals the strong influence hydration has on the membrane systems, not only on the structure but also on the dynamics. Similar to hydrated protein powders [3] also phospholipids show a dynamic transition in the mean square displacements $\left\langle u^{2}\right\rangle$ and a shift of the temperature of the main phase transition depending on their hydration. The transition for proteins occurs around $200 \mathrm{~K}$ and is often called "dynamical transition". In the case of membranes this transition is due to the structural transition into the liquidcrystalline $\mathrm{L}_{\alpha}$ phase. In contrast to proteins were the individual amino acid is hydrated to allow local motions of the proteins in phospholipid bilayers only the hydration of the hydrophilic head group of the phospholipid triggers the dynamic response of the hydration shielded hydrophobic alkyl chains. In conclusion special care should be taken for the hydration control to avoid a mixing of effects, which could be partly due to the hydration state of the sample.

\section{Acknowledgements}

We acknowledge the ILL for the allocation of beamtime and the financial support from the Access to Major Research Facilities Programme which is a component of the International Science Linkages Programme established under the Australian Government's innovation statement, Backing Australia's Ability.

\section{References}

[1] http://www.ill.eu/d16/

[2] L. Ding, T.M. Weiss, G. Fragneto, W. Liu, L. Yang and H.W. Huang, Distorted hexagonal phase studied by neutron diffraction: lipid components demixed in a bent monolayer, Langmuir 21 (2005), 203-210.

[3] W. Doster, S. Cusack and W. Petry, Dynamical transition of myoglobin revealed by inelastic neutron scattering, Nature 337 (1989), 754-756.

[4] E. Flenner, J. Das, M.C. Rheinstadter and I. Kosztin, Subdiffusion and lateral diffusion coefficient of lipid atoms and molecules in phospholipid bilayers, Physical Review E - Statistical, Nonlinear, and Soft Matter Physics 79 (2009), 011907.

[5] B. Frick and M. Gonzalez, Five years operation of the second generation backscattering spectrometer IN16 - a retrospective, recent developments and plans, Physical Review B - Condensed Matter 301 (2001), 8-19.

[6] D. Guard-Friar, C.H. Chen and A.S. Engle, Deuterium isotope effect on the stability of molecules: phospholipids, The Journal of Physical Chemistry 89 (1985), 1810-1813.

[7] S. König, W. Pfeiffer, T. Bayerl, D. Richter and E. Sackmann, Molecular dynamics of lipid bilayers studied by incoherent quasi-elastic neutron scattering, Journal de Physique II France 2 (1992), 1589-1615. 
[8] N. Kucerka, Y. Liu, N. Chu, H.I. Petrache, S. Tristram-Nagle and J.F. Nagle, Structure of fully hydrated fluid phase DMPC and DLPC lipid bilayers using X-ray scattering from oriented multilamellar arrays and from unilamellar vesicles, Biophysical Journal 88 (2005), 2626-2637.

[9] U. Lehnert, V. Réat, M. Weik, G. Zaccaï and C. Pfister, Thermal motions in bacteriorhodopsin at different hydration levels studied by neutron scattering: correlation with kinetics and light-induced conformational changes, Biophysical Journal 75 (1998), 1945-1952.

[10] J.F. Nagle, Theory of the main lipid bilayer phase transition, Annual Review of Physical Chemistry 31 (1980), 157-196.

[11] F. Natali, A. Relini, A. Gliozzi, R. Rolandi, P. Cavatorta, A. Deriu, A. Fasano and P. Riccio, The influence of the lipidprotein interaction on the membrane dynamics, Physical Review B - Condensed Matter 350 (2004), E623-E626.

[12] H. Pfeiffer, H. Binder, G. Klose and K. Heremans, Hydration pressure and phase transitions of phospholipids: I. Piezotropic approach, Biochimica et Biophysica Acta - Biomembranes 1609 (2003), 144-147.

[13] H. Pfeiffer, H. Binder, G. Klose and K. Heremans, Hydration pressure and phase transitions of phospholipids: II. Thermotropic approach, Biochimica et Biophysica Acta - Biomembranes 1609 (2003), 148-152.

[14] W. Pfeiffer, T. Henkel, E. Sackmann, W. Knoll and D. Richter, Local dynamics of lipid bilayers studied by incoherent quasi-elastic neutron scattering, Europhysics Letters 8 (1989), 201-206.

[15] M.C. Rheinstädter, C. Ollinger, G. Fragneto, F. Demmel and T. Salditt, Collective dynamics of lipid membranes studied by inelastic neutron scattering, Physical Review Letters 93 (2004), 108107.

[16] M.C. Rheinstädter, T. Seydel, F. Demmel and T. Salditt, Molecular motions in lipid bilayers studied by the neutron backscattering technique, Physical Review E - Statistical, Nonlinear, and Soft Matter Physics 71 (2005), 061908.

[17] J.H. Roh, J.E. Curtis, S. Azzam, V.N. Novikov, I. Peral, Z. Chowdhuri, R.B. Gregory and A.P. Sokolov, Influence of hydration on the dynamics of lysozyme, Biophysical Journal 91 (2006), 2573-2588.

[18] E. Sackmann, Biological membranes architecture and function, in: Structure and Dynamics of Membranes, Handbook of Biological Physics, Vol. 1, R. Lipowsky and E. Sackmann, eds, Elsevier, Amsterdam, 1995, pp. 1-64.

[19] T. Salditt and M.C. Rheinstädter, Structure and dynamics of model membrane systems probed by elastic and inelastic neutron scattering, in: Neutron Scattering in Biology, Techniques and Applications, J. Fitter, T. Gutberlet and J. Katsaras, eds, Springer, Berlin, 2006, pp. 503-530.

[20] G.S. Smith, E.B. Sirota, C.R. Safinya and N.A. Clark, Structure of the L beta phases in a hydrated phosphatidylcholine multimembrane, Physical Review Letters 60 (1988), 813-816.

[21] J.C. Smith, Protein dynamics: comparison of simulations with inelastic neutron scattering experiments, Quarterly Reviews of Biophysics 24 (1991), 227-291.

[22] J. Swenson, F. Kargl, P. Berntsen and C. Svanberg, Solvent and lipid dynamics of hydrated lipid bilayers by incoherent quasielastic neutron scattering, Journal of Chemical Physics 129 (2008), 045101.

[23] M. Trapp, F. Juranyi, M. Tehei, L. van Eijck, B. Demé, T. Gutberlet and J. Peters, QENS studies on aligned DMPC multilayers at different hydrations, in preparation. 


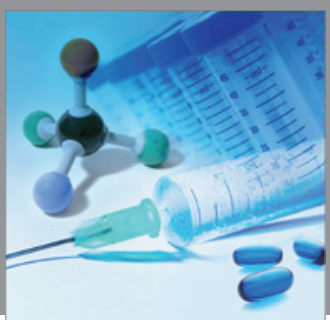

International Journal of

Medicinal Chemistry

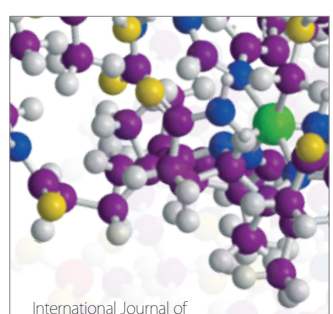

Carbohydrate Chemistry

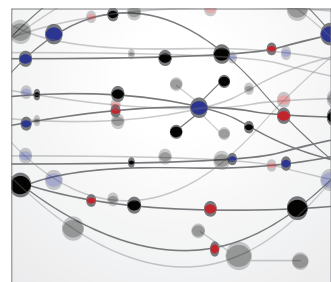

The Scientific World Journal
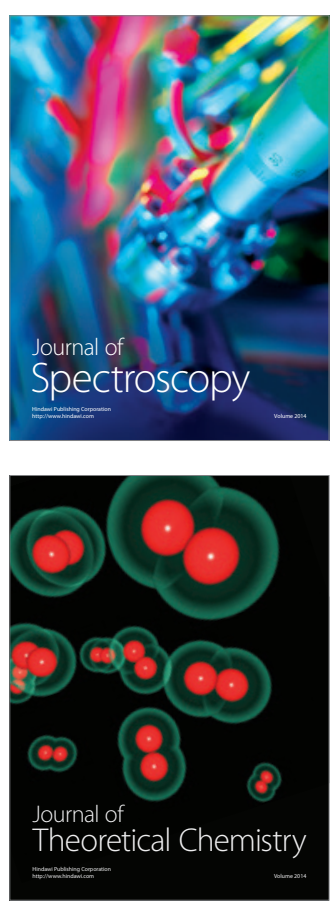
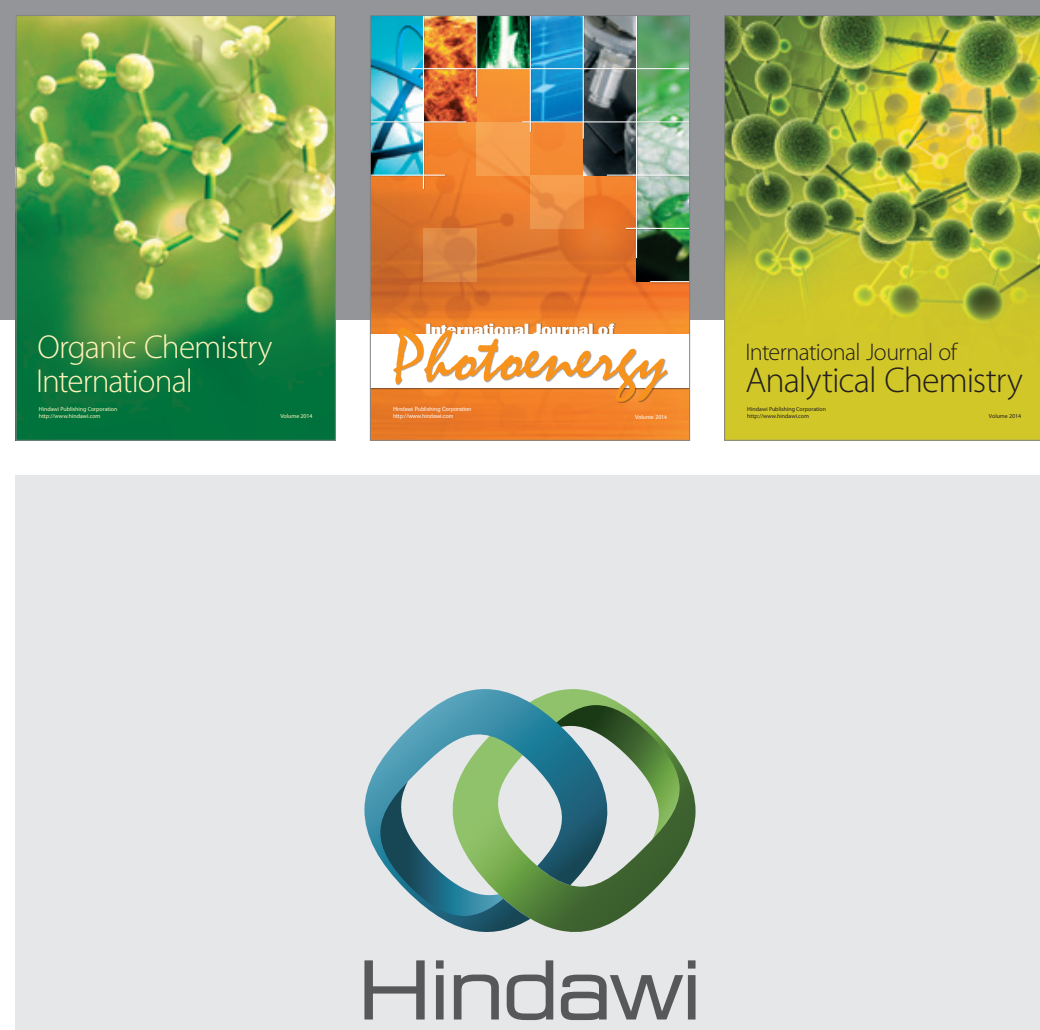

Submit your manuscripts at

http://www.hindawi.com
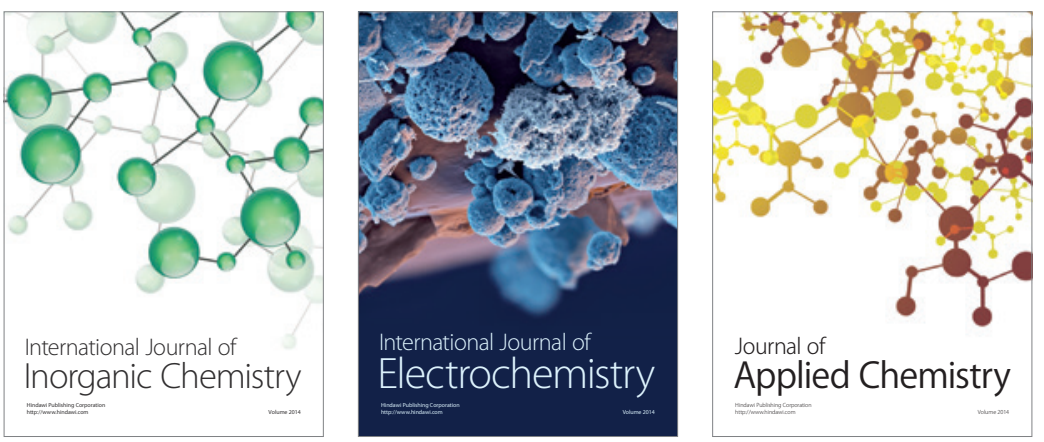

Journal of

Applied Chemistry
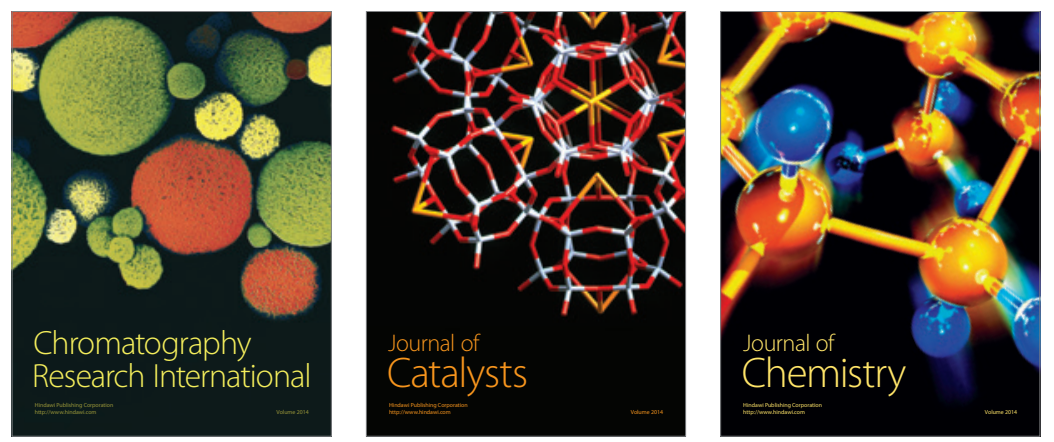
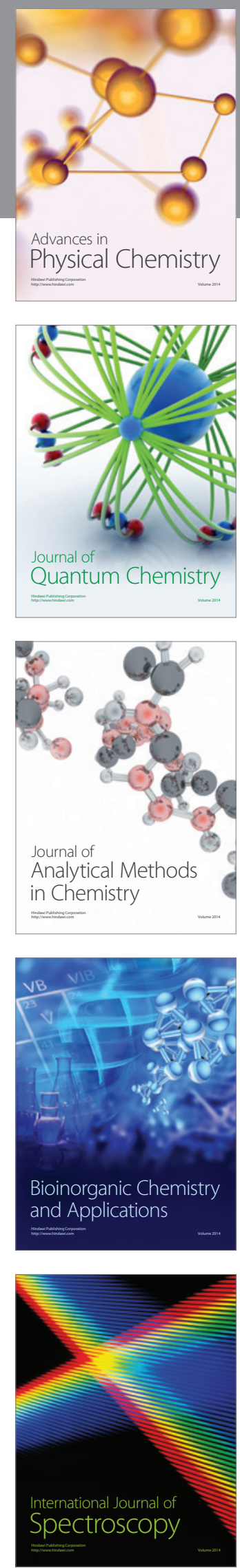